International Journal of Modern Physics A

(C) World Scientific Publishing Company

\title{
The Relativity of Chiral Property
}

\author{
Robert Delbourgo and Paul D Stack \\ School of Physical Sciences, University of Tasmania, Locked Bag 37 GPO \\ Hobart, Tasmania 7001,AUSTRALIA 7001 \\ bob.delbourgo@utas.edu.au,pdstack@utas.edu.au
}

\begin{abstract}
The standard model ascribes distinct properties to different chiralities of fermions. We show how to incorporate this aspect in an extended spacetime-property framework involving two different attributes using a generalized metric which includes gauge fields as well as gravitation. Because the gauge fields are accompanied by coupling constants, all such schemes, including ours, necessitate coupling unification at high energy to ensure universality of gravtitational interactions with matter.
\end{abstract}

\section{Chiral attributes}

The electroweak part of the standard mode $1 / 2 / 3 / 4$ unequivocally informs us that different fermion chiralities carry distinct properties, when gauged. The weak isospin $(I)$ and hypercharge $(Y)$ assignments of the chiral parts of leptonic doublets are carefully designed so that the charge $Q=I_{3}+Y / 2$ emerges correctly; however the significant point is that right and left components transform very differently under the associated gauge groups. The aim of this paper is to see how these features can be incorporated with gravity in a scheme based on spacetime coordinates augmented by Lorentz scalar anticommuting property coordinates.

In two earlier papers $\frac{566}{6 e}$ investigated such a scheme but paid scant attention to parity as we were only concerned with the unification of gravity with electromagnetism (using one property, charge) or with Yang-Mills theory (two isotopic properties). We showed how such a unification could be achieved, with the curvature arising from property. In particular, the Lagrangian and energy momentum tensor of the gauge fields that transfer property across spacetime were automatically generated. The chiral fermions were treated uniformly and assumed to have the same attributes in those works. It was clear, but more complicated, how to generalize the process for three properties, as needed in QCD, but we shied from carrying out the algebra as it led to a plethora of gauge invariant curvature coefficients in the metric that we are still uncertain how to constrain. With only two properties we have however the full machinery needed to tackle the salient properties of electroweak physics, just by focussing on the subgroup $\mathrm{U}(1)_{L} \times \mathrm{U}(1)_{Y}$, confining ourselves to the charged lepton interactions. Before doing so we shall quickly recapitulate the way this is normally handled and our unification goal, if nothing 
else to establish our conventions; this we do in Section 2. We then turn to the implementation with anticommuting scalar attributes in Section 3, starting with the fermions which are, after all, at the root of the entire gauge group; then we turn to the scalar supermultiplet, which includes the uncharged Higgs boson $\underline{7|8| 9 \mid 10}$.

The way that gravity and the gauge bosons enter is through an extended spacetime-property metric (obtained from frame vectors) which incorporates the correct gauge group, and this is described in Section 4. Having achieved the goal of combining chirality with anticommuting attributes and curved spacetime, we offer some conclusions in Section 5 and what to expect when generalizing to the full $\mathrm{SU}(3) \times \mathrm{SU}(2)_{L} \times \mathrm{U}(1)_{Y}$ of the standard model. The most significant point of our analysis is the conclusion that any scheme, such as ours, where gravity and other forces are married through some extended metric must lead to a single force strength at some large scale; extrapolation to low scales via the renormalization group has to then result in the known different forces, given the particle spectrum. Such are the overriding demands of gravitational universality.

\section{Charged leptons}

Before launching into our property scheme for dealing with chiral fermions, let us describe the endpoint attained by conventional theory, ignoring neutrinos and charged vector bosons. We are dealing therefore with the $\mathrm{U}(1)_{L} \times \mathrm{U}(1)_{Y}$ subgroup of the electroweak gauge group; this does at least include electromagnetism and the weak neutral current interactions of the charged leptons. In preserving the same charge $Q=I_{3}+Y / 2$ for the left and right pieces of the fermion field under local gauge transformations,

$$
\begin{aligned}
& \psi_{L} \rightarrow e^{-i\left(\vartheta+\vartheta^{\prime}\right) / 2} \psi_{L} \text { for } I_{3}=-1 / 2, Y=-1 \\
& \psi_{R} \rightarrow e^{-i \vartheta^{\prime}} \psi_{R} ; \quad \text { for } I=0, Y=-2,
\end{aligned}
$$

one encounters the covariant derivatives:

$$
D \psi_{L}=\left[\partial+i\left(g W+g^{\prime} B\right) / 2\right] \psi_{L}, \quad D \psi_{R}=\left[\partial+i g^{\prime} B\right] \psi_{R},
$$

where $W, B$ are of course the associated gauge fields with couplings $g, g^{\prime}$. Hence the only way to induce a mass term in addition to the usual kinetic pieces is to introduce a Higgs-like scalar $h$ which transforms inversely as

$$
h \rightarrow e^{i\left(\vartheta^{\prime}-\vartheta\right) / 2} h,
$$

so as to ensure invariance of the Yukawa interaction,

$$
\overline{\psi_{L}} h \psi_{R}+\overline{\psi_{R}} h^{\dagger} \psi_{L} .
$$

The gauge invariant kinetic motion of the scalar field,

$$
D h^{\dagger} . D h=\left[\partial+i\left(g W-g^{\prime} B\right) / 2\right] h^{\dagger} \cdot\left[\partial-i\left(g W-g^{\prime} B\right) / 2\right] h,
$$

can then provide a mass to the gauge field combination $g W-g^{\prime} B$, provided that the scalar field acquires a vacuum expectation value $\langle h\rangle=v \neq 0$, by some mechanism. 
This is where the weak mixing angle $\theta$ comes in 213; let $\tan \theta=g^{\prime} / g$ and as usual define $e=g \sin \theta=g^{\prime} \cos \theta$, whereupon the orthogonal fields $A$ and $Z$ emerge from the inverse rotation,

$$
\left(\begin{array}{l}
A \\
Z
\end{array}\right)=\left(\begin{array}{cc}
\cos \theta-\sin \theta \\
\sin \theta & \cos \theta
\end{array}\right)\left(\begin{array}{c}
B \\
W
\end{array}\right)=\frac{1}{\sqrt{g^{2}+g^{\prime 2}}}\left(\begin{array}{cc}
g & -g^{\prime} \\
g^{\prime} & g
\end{array}\right)\left(\begin{array}{c}
B \\
W
\end{array}\right)
$$

with the fermionic interaction Lagrangian for the gauge fields devolving to

$$
\begin{aligned}
\mathcal{L} & =-\overline{\psi_{L}} \gamma \cdot\left(g W+g^{\prime} B\right) \psi_{L} / 2-\overline{\psi_{R}} g^{\prime} \gamma \cdot B \psi_{R} \\
& =-e \bar{\psi} \gamma \cdot A \psi-e \cot 2 \theta \overline{\psi_{L}} \gamma \cdot Z \psi_{L}+e \tan \theta \overline{\psi_{R}} \gamma \cdot Z \psi_{R} .
\end{aligned}
$$

It is worth noting that, because $\theta \simeq 30^{\circ}$ experimentally to a rather good approximation, the interaction (at low energy) almost simplifies to

$$
\begin{aligned}
\mathcal{L} & \simeq-e \bar{\psi} \gamma \cdot A \psi+e\left(\overline{\psi_{R}} \gamma \cdot Z \psi_{R}-\overline{\psi_{L}} \gamma \cdot Z \psi_{L}\right) / \sqrt{3}=-e \bar{\psi} \gamma \cdot\left(A-i \gamma_{5} Z / \sqrt{3}\right) \psi \\
& \simeq-\left(\overline{\psi_{L}}, \overline{\psi_{R}}\right) \gamma \cdot\left(\begin{array}{cc}
e A+e^{\prime} Z & 0 \\
0 & e A-e^{\prime} Z
\end{array}\right)\left(\begin{array}{c}
\psi_{L} \\
\psi_{R}
\end{array}\right) ; \quad e^{\prime} \simeq e / \sqrt{3} .
\end{aligned}
$$

From this perspective we can view $e A$ and $e^{\prime} Z$ as forming a part of a $\mathrm{U}(2)$ group within a matrix $e A+e^{\prime} \underline{Z} \cdot \underline{\sigma}$ of which only the $Z_{3}$ component is in play. However it needs emphasizing that at the level of (7) the left and right gauge fields are not rotations amongst one another. [As we will presently see, we are forced to suppose that $e$ and $e^{\prime}$ coincide at high energy in order to respect universal gravitational coupling to matter.] It only remains to 'curve' the results (4) and (6) by including the vierbein $e_{a}^{m}(x)$ and metric $g^{m n}(x)$ into the contractions over indices and we magically get the interactions of the fermion and boson sources with gravity as well.

The job now is to try to reproduce these conventional constructs through an extended spacetime-property metric.

\section{Chiral property}

The very different behaviours of the chiral fermion components under the internal gauge transformations point to the need for distinct property coordinates; so at the very least we require two independent attributes. In an earlier paper 6 we treated the case of two properties but we assumed that they referred to 'up' and 'down' parts of an isotopic doublet, which led to the parity-conserving unification of YangMills $\mathrm{U}(2)$ with gravity; there we encountered a property curvature invariant $\bar{\zeta} \zeta \equiv$ $\bar{\zeta}_{1} \zeta^{1}+\bar{\zeta}_{2} \zeta^{2}$. Now it is no longer necessary to insist upon full $\mathrm{U}(2)$ invariance and/or parity conservation, for when we identify $\zeta^{1} \equiv \zeta_{L}$ with left-handedness and $\zeta^{2} \equiv \zeta_{R}$ with right handedness, we can permit separate $\mathrm{U}(1)_{L} \times \mathrm{U}(1)_{R}$ invariants $\overline{\zeta_{L}} \zeta_{L}$ and $\overline{\zeta_{R}} \zeta_{R}$. Our first task is to check that the flat space results can be reproduced, namely a kinetic term and no mass term, after we integrate over attribute space; the next task will be to include the scalar fields to recover induced masses and our third task will be to obtain the gravitational and gauge field interactions via an extended 
spacetime-property vielbein which 'curves' the space. At the end of the day we must recognize that the unification of parity violating interactions with gravity will not change an ugly duckling (the standard model) into a beautiful swan.

Begin with a Dirac fermion superfield $\Psi(\zeta, \bar{\zeta})$, expanded in the two chiral properties $\left(\zeta_{L}\right.$ and $\left.\zeta_{R}\right)$ which has been made anti-selfdual11 :-

$$
\begin{aligned}
2 \Psi & =\left(\overline{\zeta_{L}} \psi_{L}+\psi^{c}{ }_{L} \zeta_{L}\right)\left(1-\overline{\zeta_{R}} \zeta_{R}\right)+\left(\overline{\zeta_{R}} \psi_{R}+\psi^{c}{ }_{R} \zeta_{R}\right)\left(1-\overline{\zeta_{L}} \zeta_{L}\right) \\
& =\left(\overline{\zeta_{L}} \psi_{L}+\psi^{c}{ }_{L} \zeta_{L}+\overline{\zeta_{R}} \psi_{R}+\psi_{R}^{c} \zeta_{R}\right)(1-\bar{\zeta} \zeta) .
\end{aligned}
$$

As explained in an earlier paper, the adjoint superfield must be defined as

$$
2 \bar{\Psi}=\left(\overline{\zeta_{L} \psi_{L}^{c}}-\overline{\psi_{L}} \zeta_{L}+\overline{\zeta_{R} \psi_{R}^{c}}-\overline{\psi_{R}} \zeta_{R}\right)(1-\bar{\zeta} \zeta) .
$$

Because of spinorial orthogonality this means that a mass-like term,

$$
2 \bar{\Psi} \Psi=\overline{\zeta_{R}} \zeta_{L} \overline{\psi_{L}} \psi_{R}+(L \leftrightarrow R),
$$

will automatically give zero when integrated over property space as there are insufficient numbers of $\zeta$ s. However a kinetic term,

$$
2 \bar{\Psi} i \gamma . \partial \Psi=\overline{\zeta_{L}} \zeta_{L}\left(1-2 \overline{\zeta_{R}} \zeta_{R}\right) \overline{\psi_{L}} i \gamma \cdot \partial \psi_{L}+(L \leftrightarrow R),
$$

is perfectly fine as it survives integration over property. It is very encouraging that we require an extra scalar superfield to induce a mass interaction since it reverberates with gauge invariance requirements.

Thus consider the real anti-selfdual superscalar :

$$
\Phi(\zeta, \bar{\zeta})=\phi\left(1-\overline{\zeta_{R}} \zeta_{R} \overline{\zeta_{L}} \zeta_{L}\right)+h \overline{\zeta_{L}} \zeta_{R}+h^{\dagger} \overline{\zeta_{R}} \zeta_{L}+\varphi\left(\overline{\zeta_{L}} \zeta_{L}-\overline{\zeta_{R}} \zeta_{R}\right)
$$

When interacting with $\Psi$, this will include a piece

$$
\bar{\Psi} \Phi \Psi \supset \overline{\zeta_{R}} \zeta_{L} \overline{\psi_{L}} \psi_{R} h \overline{\zeta_{L}} \zeta_{R}+\text { h.c. }
$$

A vacuum expectation value for the chargeless field $\langle h\rangle$ does duty for a mass term; it will also make an appropriate mixture of gauge bosons massive when we introduce curvature into property space.

\section{Gauge and Gravity extended metric}

The most important objective is to get the correct coupling of gauge fields and gravity to the chiral fermions through a generalized spacetime-property metric. The rest (coupling to bosons, gauge-gravity Lagrangian, etc.) will take care of itself. In a previous paper $\underline{6}$, given two properties, we proved that with a metric field $G_{M N}$ in full $x-\zeta$ space having components

$$
\begin{aligned}
G_{m n} & =g_{m n}\left[1+c_{1} \bar{\zeta} \zeta+c_{2}(\bar{\zeta} \zeta)^{2}\right]+e^{2} l^{2} \bar{\zeta}\left(W_{m} W_{n}+W_{n} W_{m}\right) \zeta\left(1+c_{3} \bar{\zeta} \zeta\right) / 2, \\
G_{m \nu} & =i e l^{2}\left(\bar{\zeta} W_{m}\right)^{\bar{\nu}}\left(1+c_{3} \bar{\zeta} \zeta\right) / 2, \quad \text { etc. }
\end{aligned}
$$


we could reproduce all gravitational plus gauge field interactions reliably via the total superscalar curvature $\mathcal{R}$, possessing full $\mathrm{U}(2)$ invariance. Such a metric resulted from frame vectors with components

$$
\mathcal{E}_{m}{ }^{\alpha}=-i e\left(1+c_{3} \bar{\zeta} \zeta / 2\right)\left(W_{m} \zeta\right)^{\alpha}, \quad \mathcal{E}_{m}{ }^{\bar{\alpha}}=i e\left(1+c_{3} \bar{\zeta} \zeta / 2\right)\left(\bar{\zeta} W_{m}\right)^{\bar{\alpha}}, \quad \text { etc. }
$$

In our case we do not require full $\mathrm{U}(2)$ invariance but merely $\mathrm{U}(1) \times \mathrm{U}(1)$ symmetry, as dictated by experiment for charged leptons; so we can relax the condition that the curvature coefficients $c$ and the couplings be the same for the two associated gauge fields $A$ and $Z$. We have a lot more freedom in constructing the curvatures because $\bar{\zeta}_{L} \zeta_{L}$ and $\bar{\zeta}_{R} \zeta_{R}$ are separately invariant. [The appendix contains a full discussion of all property curvatures that do not disturb the $\mathrm{U}(1) \times \mathrm{U}(1)$ symmetry and the complications that ensue.] In particular the vielbeins $E_{a}{ }^{\zeta_{L}}, E_{a}{ }^{{ }}$given there have forms which ensure that the couplings of the gauge fields to the fermions correctly sum to $e_{L} \bar{\psi}_{L} \gamma \cdot W_{L} \psi_{L}+e_{R} \bar{\psi}_{R} \gamma \cdot W_{R} \psi_{R}$. Then reinterpreting $e_{L} W_{L}=e A+e^{\prime} Z$, $e_{R} W_{R}=e A-e^{\prime} Z$, we can indeed reproduce (7). This is all well and good, but we shall presently see that universal gravitational coupling to the stress tensors of the gauge fields imposes a strong constraint on the curvature coefficients.

The appendix contains a full treatment of all possible property curvatures, consistent with the gauge symmetry. Identifying $c_{4}$ with $c_{L}$ and $c_{5}$ with $c_{R}$, the metric $G_{m \nu}$ in the $x-\zeta$ sector equals

$$
-i\left[l^{2} \bar{\zeta}_{L} e_{L} W_{L}\left(1+c_{L} \bar{\zeta} \zeta\right)+\ell^{2} e_{R} W_{R}\left(1+c_{R} \bar{\zeta} \zeta\right)\right] / 2 .
$$

Under the two independent local coordinate transformations,

$$
\zeta_{L} \rightarrow \zeta_{L}^{\prime}=e^{i \theta_{L}} \zeta_{L}, \quad \zeta_{R} \rightarrow \zeta_{R}^{\prime}=e^{i \theta_{R}} \zeta_{R},
$$

it is readily checked, from the rules of transformation of the metric tensor, that these simply correspond to independent gauge transformations,

$$
e_{L} W_{L}^{\prime}=e_{L} W_{L}+\partial \theta_{L}, \quad e_{R} W_{R}^{\prime}=e_{R} W_{R}+\partial \theta_{R} .
$$

In turn this translates into

$$
e A \rightarrow e A+\partial \theta, \quad e^{\prime} Z \rightarrow e^{\prime} Z+\partial \theta^{\prime},
$$

which are the independent gauge variations of the more standard gauge fields $A$ and $Z$. However we are faced with a serious problem when we introduce gravitation...

The dilemma which confronts us is best seen by working out the integral over the superscalar curvature $\mathcal{R}$, as summarized in the appendix:

$$
\mathcal{L}=\int d^{2} \zeta d^{2} \bar{\zeta} \sqrt{-G . .} \mathcal{R}=\sqrt{-g . .}\left[\frac{4 B R^{[g]}}{l^{2} \ell^{2}}-\frac{e_{L}^{2} c_{8}}{\ell^{2}} F_{L}^{m n} F_{L m n}-\frac{e_{R}^{2} c_{6}}{l^{2}} F_{R}^{m n} F_{R m n}+C\right]
$$

where $C$ denotes a cosmological term, $F_{m n} \equiv W_{n, m}-W_{m, n}$ and $R^{[g]}$ is the purely gravitational scalar curvature. If we then make the replacements

$$
e_{L} W_{L}=e A+e^{\prime} Z, \quad e_{R} W_{R}=e A-e^{\prime} Z,
$$


we need to ensure cancellation of the cross $A Z$ term which, in turn, forces $c_{8} / \ell^{2}=$ $c_{6} / l^{2}$. Finally the gauge field Lagrangians have to couple universally to gravity through their stress tensors so we must further set $e^{\prime}=e$ as a proper normalization condition. This is a very strong consequence of attaching force coupling constants to gauge fields in the metric itself.

\section{Concluding Remarks}

This need for coupling constant equality seems to be an unwelcome result. But a moment's reflection leads one to the conclusion that it is an inescapable feature which arises from merging the gauge fields and gravity in some kind of enlarged metric, not just in our scheme but in any other scheme based on the same concepts. The significant point is that at a semiclassical leve the coupling constants must always accompany the gauge fields, via the frame vectors that latch on to the fermions, and this baggage is inevitably carried into the stress tensors that emerge from $G$.

So the question arises: Is this an impasse or not? We would suggest, like many others, that the couplings may very well be equal (or are unified) at some high energy scale $l \simeq 10^{13} \mathrm{GeV}$ or more, associated with $l$, signifying $e^{2}(l)=e^{\prime 2}(l)$, but that the observed values at low energy $e^{\prime} \simeq e / \sqrt{3}$ are due to running down in energy to laboratory scales (corresponding to different gauge groups with their particle contents). We would go so far as to assert that any theory which attempts to unify gravity with the other forces faces the same dilemma and the only reasonable solution is to demand equality of all coupling constants at Planckian type scales. The corollary is that if the continuation of the constants to low energy via the renormalization group does not match experiment, either the particle content associated with the gauge group is wrong or, more drastically, the extended metric theory is doomed, unless it is regarded as fully quantum and contains the chargelike operators within the frame vectors $\mathrm{b}$.

\section{Appendix A. Extended Chiral Minkowski Metric}

We extend spacetime with two property coordinates, $\zeta_{L}$ and $\zeta_{R}$. This gives superspace coordinates of $X^{M}=\left(x^{m}, \zeta_{L}, \zeta_{R}, \bar{\zeta}_{L}, \bar{\zeta}_{R}\right)$. Our starting point to building the metric is the following metric distance for a flat $4+4$ dimensional graded manifold:

$d s^{2}=d X^{A} d X^{B} \mathcal{I}_{B A}=d x^{a} d x^{b} \eta_{b a}+\frac{1}{2} l^{2} d \zeta_{L} d \overline{\zeta_{L}}-\frac{1}{2} l^{2} d \overline{\zeta_{L}} d \zeta_{L}+\frac{1}{2} \ell^{2} d \zeta_{R} d \overline{\zeta_{R}}-\frac{1}{2} \ell^{2} d \overline{\zeta_{R}} d \zeta_{R}$

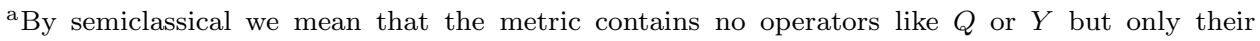
eigenvalues such as $e$ or $e^{\prime}$

${ }^{b}$ We have never before seen the incorporation of differential operators of the coordinates even in a quantized metric field.
} 
This results in the extended Minkowski metric $\mathcal{I}_{A B}$ taking the form:

$$
\mathcal{I}_{A B}=\left(\begin{array}{ccccc}
I_{a b} & I_{a \zeta_{L}} & I_{a \zeta_{R}} & I_{a \bar{\zeta}_{L}} & I_{a \bar{\zeta}_{R}} \\
I_{\zeta_{L} b} & I_{\zeta_{L} \zeta_{L}} & I_{\zeta_{L} \zeta_{R}} & I_{\zeta_{L} \bar{\zeta}_{L}} & I_{\zeta_{L} \bar{\zeta}_{R}} \\
I_{\zeta_{R} b} & I_{\zeta_{R} \zeta_{L}} & I_{\zeta_{R} \zeta_{R}} & I_{\zeta_{R} \bar{\zeta}_{L}} & I_{\zeta_{R} \bar{\zeta}_{R}} \\
I_{\bar{\zeta}_{L} b} & I_{\bar{\zeta}_{L} \zeta_{L}} & I_{\bar{\zeta}_{L} \zeta_{R}} & I_{\bar{\zeta}_{L} \bar{\zeta}_{L}} & I_{\bar{\zeta}_{L} \bar{\zeta}_{R}} \\
I_{\bar{\zeta}_{R} b} & I_{\bar{\zeta}_{R} \zeta_{L}} & I_{\bar{\zeta}_{R} \zeta_{R}} & I_{\bar{\zeta}_{R} \bar{\zeta}_{L}} & I_{\bar{\zeta}_{R} \bar{\zeta}_{R}}
\end{array}\right)=\left(\begin{array}{ccccc}
\eta_{a b} & 0 & 0 & 0 & 0 \\
0 & 0 & 0 & \frac{1}{2} l^{2} & 0 \\
0 & 0 & 0 & 0 & \frac{1}{2} \ell^{2} \\
0 & -\frac{1}{2} l^{2} & 0 & 0 & 0 \\
0 & 0 & -\frac{1}{2} \ell^{2} & 0 & 0
\end{array}\right)
$$

Notice that we have introduced two separate length scales $l$ and $\ell$ in order to give ourselves complete freedom and wriggle room.

\section{Appendix B. Frame vectors and Gauge fields}

Consider spacetime dependent $\mathrm{U}(1) \times \mathrm{U}(1)$ phase transformations to the property coordinates $\zeta_{L}$ and $\zeta_{R}$ as follows:

$x^{m} \rightarrow x^{m}, \quad \zeta_{L} \rightarrow e^{i \theta_{L}(x)} \zeta_{L}, \quad \bar{\zeta}_{L} \rightarrow e^{-i \theta_{L}(x)} \zeta_{L}, \quad \zeta_{R} \rightarrow e^{i \theta_{R}(x)} \zeta_{R}, \quad \bar{\zeta}_{R} \rightarrow e^{-i \theta_{R}(x)} \bar{\zeta}_{R}$.

Now if we want our metric $\mathcal{I}_{A B}$ to be a tensor it has to transform correctly. With $x$ dependent phase changes in property it does not. Therefore, as in the one coordinate case, we need to fix this by including gauge fields. We do so by introducing the following upper-triangular frame vector:

$$
\mathcal{E}_{M}{ }^{A}=\left(\begin{array}{ccccc}
e_{m}{ }^{a}-i L_{m} \zeta_{L} & -i R_{m} \zeta_{R} & i \bar{\zeta}_{L} L_{m} & i \bar{\zeta}_{R} R_{m} \\
0 & 1 & 0 & 0 & 0 \\
0 & 0 & 1 & 0 & 0 \\
0 & 0 & 0 & 1 & 0 \\
0 & 0 & 0 & 0 & 1
\end{array}\right)
$$

and its inverse or the vielbein (with $\mathcal{E}_{\mathcal{M}}{ }^{A} E_{A}{ }^{N}=\delta_{M}{ }^{N}$ ):

$$
E_{A}{ }^{M}=\left(\begin{array}{ccccc}
e_{a}{ }^{m} & i L_{a} \zeta_{L} & i R_{a} \zeta_{R}-i \bar{\zeta}_{L} L_{a} & -i \bar{\zeta}_{R} R_{a} \\
0 & 1 & 0 & 0 & 0 \\
0 & 0 & 1 & 0 & 0 \\
0 & 0 & 0 & 1 & 0 \\
0 & 0 & 0 & 0 & 1
\end{array}\right)
$$

whereupon the metric follows from $G_{M N}=(-1)^{A N} \mathcal{E}_{M}{ }^{A} \mathcal{E}_{N}{ }^{B} \mathcal{I}_{B A}$; explicitly we get,

$G_{M N}=\left(\begin{array}{ccccc}g_{m n}+l^{2} L_{m} L_{n} \bar{\zeta}_{L} \zeta_{L}+\ell^{2} R_{m} R_{n} \bar{\zeta}_{R} \zeta_{R}-\frac{1}{2} i l^{2} \bar{\zeta}_{L} L_{m} & -\frac{1}{2} i \ell^{2} \bar{\zeta}_{R} R_{m} & -\frac{1}{2} i l^{2} L_{m} \zeta_{L}-\frac{1}{2} i \ell^{2} R_{m} \zeta_{R} \\ -\frac{1}{2} i l^{2} \bar{\zeta}_{L} L_{m} & 0 & 0 & l^{2} / 2 & 0 \\ -\frac{1}{2} i \ell^{2} \bar{\zeta}_{R} R_{m} & 0 & 0 & 0 & \ell^{2} / 2 \\ -\frac{1}{2} i l^{2} L_{m} \zeta_{L} & -l^{2} / 2 & 0 & 0 & 0 \\ -\frac{1}{2} i \ell^{2} R_{m} \zeta_{R} & 0 & -\ell^{2} / 2 & 0 & 0\end{array}\right)$.

It is readily checked from the tensorial transformation of the metric that the changes (B.1) correctly yield the gauge variations,

$$
L_{m}^{\prime}=L_{m}+\theta_{L, m}, \quad R_{m}^{\prime}=R_{m}+\theta_{R, m} .
$$


[Note, there is no need to include gauge couplings $e_{R}$ and $e_{L}$ with the gauge fields $R$ and $L$ at this stage. In the text $R$ and $L$ have been replaced by $e_{R} W_{R}$ and $e_{L} W_{L}$ .]

\section{Appendix C. Curvature invariants}

Representation (B.4) is not the end of the story. We have the freedom to include terms in the metric which are $\mathrm{U}(1) \times \mathrm{U}(1)$ invariant, involving $\bar{\zeta}_{L} \zeta_{L}, \bar{\zeta}_{R} \zeta_{R}$. But in so doing we have to ensure that all components of $G$ transform correctly; after long and careful analysis we find that the nonzero metric elements can be finally reduced to

$$
\begin{aligned}
G_{m n} & =g_{m n}\left[1+c_{1} \bar{\zeta}_{L} \zeta_{L}+c_{2} \bar{\zeta}_{R} \zeta_{R}+c_{3} \bar{\zeta}_{L} \zeta_{L} \bar{\zeta}_{R} \zeta_{R}\right]+l^{2} L_{m} L_{n} \bar{\zeta}_{L} \zeta_{L}\left(1+c_{4} \bar{\zeta}_{R} \zeta_{R}\right)+\ell^{2} R_{m} R_{n} \bar{\zeta}_{R} \zeta_{R}\left(1+c_{5} \bar{\zeta}_{L} \zeta_{L}\right) \\
G_{m \zeta_{L}} & =-i l^{2} \bar{\zeta}_{L} L_{m}\left(1+c_{4} \bar{\zeta}_{R} \zeta_{R}\right) / 2 \\
G_{m \zeta_{R}} & =-i \ell^{2} \bar{\zeta}_{R} R_{m}\left(1+c_{5} \bar{\zeta}_{L} \zeta_{L}\right) / 2 \\
G_{m \bar{\zeta}_{L}} & =-i l^{2} L_{m} \zeta_{L}\left(1+c_{4} \bar{\zeta}_{R} \zeta_{R}\right) / 2 \\
G_{m \bar{\zeta}_{R}} & =-i \ell^{2} R_{m} \zeta_{R}\left(1+c_{5} \bar{\zeta}_{L} \zeta_{L}\right) / 2 \\
G_{\zeta_{L} \bar{\zeta}_{L}} & =l^{2}\left(1+c_{6} \bar{\zeta}_{L} \zeta_{L}+c_{4} \bar{\zeta}_{R} \zeta_{R}+c_{7} \bar{\zeta}_{L} \zeta_{L} \bar{\zeta}_{R} \zeta_{R}\right) / 2 \\
G_{\zeta_{R} \bar{\zeta}_{R}} & =\ell^{2}\left(1+c_{5} \bar{\zeta}_{L} \zeta_{L}+c_{8} \bar{\zeta}_{R} \zeta_{R}+c_{9} \bar{\zeta}_{L} \zeta_{L} \bar{\zeta}_{R} \zeta_{R}\right) / 2
\end{aligned}
$$

The ensuing inverse metric components read:

$$
\begin{aligned}
G^{m n} & =g^{m n}\left[1-c_{1} \bar{\zeta}_{L} \zeta_{L}-c_{2} \bar{\zeta}_{R} \zeta_{R}+\left(2 c_{1} c_{2}-c_{3}\right) \bar{\zeta}_{L} \zeta_{L} \bar{\zeta}_{R} \zeta_{R}\right], \\
G^{m \zeta_{L}} & =i L^{m} \zeta_{L}\left(1-c_{2} \bar{\zeta}_{R} \zeta_{R}\right), \\
G^{m \zeta_{R}} & =i R^{m} \zeta_{R}\left(1-c_{1} \bar{\zeta}_{L} \zeta_{L}\right), \\
G^{m \bar{\zeta}_{L}} & =-i L^{m} \bar{\zeta}_{L}\left(1-c_{2} \bar{\zeta}_{R} \zeta_{R}\right), \\
G^{m \bar{\zeta}_{R}} & =-i R^{m} \bar{\zeta}_{R}\left(1-c_{1} \bar{\zeta}_{L} \zeta_{L}\right), \\
G^{\zeta_{L} \bar{\zeta}_{L}} & =2\left[1-c_{6} \bar{\zeta}_{L} \zeta_{L}-c_{4} \bar{\zeta}_{R} \zeta_{R}+\left(2 c_{6} c_{4}-c_{7}\right) \bar{\zeta}_{L} \zeta_{L} \bar{\zeta}_{R} \zeta_{R}\right] / l^{2}-L^{m} L_{m} \bar{\zeta}_{L} \zeta_{L}\left(1-c_{2} \bar{\zeta}_{R} \zeta_{R}\right), \\
G^{\zeta_{R} \bar{\zeta}_{R}} & =2\left[1-c_{5} \bar{\zeta}_{L} \zeta_{L}-c_{8} \bar{\zeta}_{R} \zeta_{R}+\left(2 c_{5} c_{8}-c_{9}\right) \bar{\zeta}_{L} \zeta_{L} \bar{\zeta}_{R} \zeta_{R}\right] / \ell^{2}-R^{m} R_{m} \bar{\zeta}_{R} \zeta_{R}\left(1-c_{1} \bar{\zeta}_{L} \zeta_{L}\right), \\
G^{\zeta_{L} \zeta_{R}} & =-L^{m} R_{m} \zeta_{L} \zeta_{R}, \\
G^{\bar{\zeta}_{L} \bar{\zeta}_{R}} & =-L^{m} R_{m} \bar{\zeta}_{L} \bar{\zeta}_{R} \\
G^{\zeta_{L} \bar{\zeta}_{R}} & =L^{m} R_{m} \zeta_{L} \bar{\zeta}_{R}, \\
G^{\zeta_{R} \bar{\zeta}_{L}} & =L^{m} R_{m} \zeta_{R} \bar{\zeta}_{L} .
\end{aligned}
$$

In the text we have substituted $c_{4}$ by $c_{L}$ and $c_{5}$ by $c_{R}$; on the other hand, $c_{8}, c_{6}$ enter the normalization of the Lagrangians for the left and right gauge fields separately. 


\section{Appendix D. Metric superdeterminant}

The Berezinian or superdeterminant requires some more work. Following standard procedures the superdeterminant is obtained from the graded supermatrix,

$$
\begin{aligned}
\sqrt{G_{. .}} & =\frac{4 \sqrt{g}}{l^{2} \ell^{2}}\left[1+\left(2 c_{1}-c_{5}-c_{6}\right) \bar{\zeta} \zeta+\left(2 c_{2}-c_{4}-c_{8}\right) \bar{\zeta}_{R} \zeta_{R}\right. \\
& \left.+\left(2 c_{1} c_{2}+2 c_{3}-2 c_{1} c_{4}-2 c_{2} c_{5}+c_{4} c_{5}-2 c_{2} c_{6}+2 c_{4} c_{6}-c_{7}-2 c_{1} c_{8}+2 c_{5} c_{8}+c_{6} c_{8}-c_{9}\right) \bar{\zeta}_{L} \zeta_{L} \bar{\zeta}_{R} \zeta_{R}\right] .
\end{aligned}
$$

The frame vectors $\mathcal{E}_{M}{ }^{A}$ which produce the metric have the components:

$$
\begin{aligned}
\mathcal{E}_{m}{ }^{a} & =e_{m}{ }^{a}\left[1+\frac{c_{1}}{2} \bar{\zeta}_{L} \zeta_{L}+\frac{c_{2}}{2} \bar{\zeta}_{R} \zeta_{R}+\left(\frac{c_{3}}{2}-\frac{c_{1} c_{2}}{4}\right) \bar{\zeta}_{L} \zeta_{L} \bar{\zeta}_{R} \zeta_{R}\right], \\
\mathcal{E}_{m}{ }^{\zeta_{L}} & =-i L_{m} \zeta_{L}\left(1+c_{4} \bar{\zeta}_{R} \zeta_{R} / 2\right), \\
\mathcal{E}_{m}{ }^{\zeta_{R}} & =-i R_{m} \zeta_{R}\left(1+c_{5} \bar{\zeta}_{L} \zeta_{L} / 2\right), \\
\mathcal{E}_{m} \bar{\zeta}_{L} & =i L_{m} \bar{\zeta}_{L}\left(1+c_{4} \bar{\zeta}_{R} \zeta_{R} / 2\right), \\
\mathcal{E}_{m}{ }^{\bar{\zeta}_{R}} & =i R_{m} \bar{\zeta}_{R}\left(1+c_{5} \bar{\zeta}_{L} \zeta_{L} / 2\right), \\
\mathcal{E}_{\zeta_{L}}{ }^{\zeta_{L}} & =\mathcal{E}_{\bar{\zeta}_{L}} \bar{\zeta}_{L}=1+\frac{c_{6}}{2} \bar{\zeta}_{L} \zeta_{L}+\frac{c_{4}}{2} \bar{\zeta}_{R} \zeta_{R}+\left(\frac{c_{7}}{2}-\frac{c_{6} c_{4}}{4}\right) \bar{\zeta}_{L} \zeta_{L} \bar{\zeta}_{R} \zeta_{R}, \\
\mathcal{E}_{\zeta_{R}}{ }^{\zeta_{R}} & =\mathcal{E}_{\bar{\zeta}_{R}} \bar{\zeta}_{R}=1+\frac{c_{5}}{2} \bar{\zeta}_{L} \zeta_{L}+\frac{c_{8}}{2} \bar{\zeta}_{R} \zeta_{R}+\left(\frac{c_{9}}{2}-\frac{c_{5} c_{8}}{4}\right) \bar{\zeta}_{L} \zeta_{L} \bar{\zeta}_{R} \zeta_{R} .
\end{aligned}
$$

Also needed are:

$$
\begin{aligned}
\left(\mathcal{E}_{\zeta_{L}}^{\zeta_{L}}\right)^{-1} & =\left(\mathcal{E}_{\bar{\zeta}_{L}} \bar{\zeta}_{L}\right)^{-1}=1-\frac{c_{6}}{2} \bar{\zeta}_{L} \zeta_{L}-\frac{c_{4}}{2} \bar{\zeta}_{R} \zeta_{R}-\left(\frac{c_{7}}{2}-\frac{3 c_{6} c_{4}}{4}\right) \bar{\zeta}_{L} \zeta_{L} \bar{\zeta}_{R} \zeta_{R} \\
\left(\mathcal{E}_{\zeta_{R}}{ }^{\xi}\right)^{-1} & =\left(\mathcal{E}_{\bar{\zeta}_{R}} \bar{\zeta}_{R}\right)^{-1}=1-\frac{c_{5}}{2} \bar{\zeta}_{L} \zeta_{L}-\frac{c_{8}}{2} \bar{\zeta}_{R} \zeta_{R}-\left(\frac{c_{9}}{2}-\frac{3 c_{5} c_{8}}{4}\right) \bar{\zeta}_{L} \zeta_{L} \bar{\zeta}_{R} \zeta_{R}
\end{aligned}
$$

As a useful crosscheck, $\operatorname{sdet} \mathcal{E}=\sqrt{G_{. .}} / \sqrt{\operatorname{sdet} I}$, so all is as it should be.

\section{Appendix E. The superscalar curvature}

Now to find the Lagrangian. Using the Palatini form of the Ricci scalar ${ }^{[5}$ we get:

$$
\mathcal{L}=\int \sqrt{G_{. .}} \mathcal{R} d \zeta_{L} d \bar{\zeta}_{L} d \zeta_{R} d \bar{\zeta}_{R}=\frac{4 B}{l^{2} \ell^{2}} R^{[g]}-\frac{c_{6}}{l^{2}} R^{m n} R_{m n}-\frac{c_{8}}{\ell^{2}} L^{m n} L_{m n}+C,
$$

where $R_{m n} \equiv R_{n, m}-R_{m, n}, \quad L_{m n} \equiv L_{n, m}-L_{m, n}$,

$$
B=c_{7}+c_{9}-c_{3}+c_{1} c_{4}+c_{2} c_{5}-c_{4} c_{5}+c_{2} c_{6}-2 c_{4} c_{6}+c_{1} c_{8}-2 c_{5} c_{8}-c_{6} c_{8}
$$

and

$C=\frac{16}{l^{4} \ell^{4}}\left[-6 \ell^{2} c_{1} c_{3}-6 l^{2} c_{2} c_{3}+6 \ell^{2} c_{1}^{2} c_{4}+4 l^{2} c_{1} c_{2} c_{4}+4 l^{2} c_{3} c_{4}-3 l^{2} c_{1} c_{4}^{2}+4 \ell^{2} c_{1} c_{2} c_{5}+\right.$ $6 l^{2} c_{2}^{2} c_{5}+4 \ell^{2} c_{3} c_{5}-8 \ell^{2} c_{1} c_{4} c_{5}-8 l^{2} c_{2} c_{4} c_{5}+3 l^{2} c_{4}^{2} c_{5}-3 \ell^{2} c_{2} c_{5}^{2}+3 \ell^{2} c_{4} c_{5}^{2}+6 \ell^{2} c_{1} c_{2} c_{6}+$ $3 l^{2} c_{2}^{2} c_{6}+6 \ell^{2} c_{3} c_{6}-18 \ell^{2} c_{1} c_{4} c_{6}-8 l^{2} c_{2} c_{4} c_{6}+\frac{9}{2} l^{2} c_{4}^{2} c_{6}-6 \ell^{2} c_{2} c_{5} c_{6}+9 \ell^{2} c_{4} c_{5} c_{6}-6 \ell^{2} c_{2} c_{6}^{2}+$ $12 \ell^{2} c_{4} c_{6}^{2}+6 \ell^{2} c_{1} c_{7}+4 l^{2} c_{2} c_{7}-3 l^{2} c_{4} c_{7}-3 \ell^{2} c_{5} c_{7}-6 \ell^{2} c_{6} c_{7}+3 \ell^{2} c_{1}^{2} c_{8}+6 l^{2} c_{1} c_{2} c_{8}+$ 
$6 l^{2} c_{3} c_{8}-6 l^{2} c_{1} c_{4} c_{8}-8 \ell^{2} c_{1} c_{5} c_{8}-18 l^{2} c_{2} c_{5} c_{8}+9 l^{2} c_{4} c_{5} c_{8}+\frac{9}{2} \ell^{2} c_{5}^{2} c_{8}-6 \ell^{2} c_{1} c_{6} c_{8}-$ $6 l^{2} c_{2} c_{6} c_{8}+6 l^{2} c_{4} c_{6} c_{8}+6 \ell^{2} c_{5} c_{6} c_{8}+3 \ell^{2} c_{6}^{2} c_{8}-3 l^{2} c_{7} c_{8}-6 l^{2} c_{1} c_{8}^{2}+12 l^{2} c_{5} c_{8}^{2}+3 l^{2} c_{6} c_{8}^{2}+$ $\left.4 \ell^{2} c_{1} c_{9}+6 l^{2} c_{2} c_{9}-3 l^{2} c_{4} c_{9}-3 \ell^{2} c_{5} c_{9}-3 \ell^{2} c_{6} c_{9}-6 l^{2} c_{8} c_{9}\right]$.

This is all very complicated but it demonstrates how many gauge invariant property curvature terms work their way into the system -9 in our case. One therefore needs some guiding principle for cutting them down. One possible suggestion is to make all the curvature pieces fully $\mathrm{U}(n)$ invariant with $n$ properties, except for those products that involve the gauge fields embedded in the frame vectors; so in our instance one could think of setting

$$
\ell=l, \quad c_{1}=c_{2}, \quad c_{4}=c_{5}=c_{6}=c_{8}, \quad c_{7}=c_{9},
$$

leaving us with only four constant: $\mathrm{C}$, one scale and one coupling constant. Although this simplification really needs some fundamental justification, it can be used to make the four-property case of $\mathrm{SU}(2)_{L} \times \mathrm{U}(1)$ more manageable and is even extensible to the direct product with chromodynamics. The main point is this: the property invariant curvatures must be severely constrained before one can handle the full standard model; we are uncertain how to do so at this stage.

\section{References}

1. S.L Glashow, Nucl. Phys. 22, 579 (1961).

2. A. Salam and J.C. Ward, Phys. Lett. 13,168 (1964).

3. S. Weinberg, Phys. Fev. Lett. 19,1264 (1967).

4. A. Salam, Eighth Nobel Symposium, ed. N. Svartholm, Almquist and Wiksell, (1968).

5. R. Delbourgo and P.D. Stack, Int. J. Mod. Phys. 29A, 50023 (2014).

6. P.D. Stack and R. Delbourgo, Int. J. Mod. Phys. 30A,1550005 (2015).

7. F. Englert and R. Brout, Phys. Rev. Lett. 13, 321 (1964).

8. P.W. Higgs, Phys. Rev.Lett. 13, 508 (1964).

9. G.S. Guralnik, C.R. Hagen and T.W.B. Kibble, Phys. Rev. Lett. 13, 585 (1964).

10. T.W.B. Kibble, Phys. Rev. 155, 1554 (1967).

11. R. Delbourgo, J. Phys. A39, 14735 (2006).

${ }^{\mathrm{c}}$ Indeed if we were to set $c_{4}=c_{1}, c_{7}=c_{3} \equiv c_{1}^{2} b_{3}$, we could reduce the whole down to $\Lambda c^{4} / 8 \pi G_{N}=$ $8 c_{1}^{2} b_{3} / l^{4} e^{2}, 8 \pi G_{N} / c^{4}=2 l^{2} e^{2} / c_{1}\left(b_{3}-2\right)$ and there would just be an overall property factor [1+ $\left.c_{1} \bar{\zeta} \zeta+2\left(c_{1} \bar{\zeta} \zeta\right)^{2}\right]$ multiplying the property flat elements, including the gauge field elements $W \zeta$ in the $x-\zeta$ sector. 